\title{
Analytical Methodologies for the Determination of Biogenic Amines in Wines: An Overview of the Recent Trends
}

\author{
Andreia Miranda ${ }^{1,2}$, João M. Leça ${ }^{1,2}$, Vanda Pereira ${ }^{1,2^{*}}$, José C. Marques ${ }^{1,2}$ \\ ${ }^{1}$ Faculty of Exact Sciences and Engineering, University of Madeira, Campus da Penteada, 9020-105 Funchal, Portugal \\ ${ }^{2}$ Institute of Nanostructures, Nanomodelling and Nanofabrication (I3N), University of Aveiro, Aveiro, Portugal
}

*Corresponding author: Vanda Pereira, Faculty of Exact Sciences and Engineering, University of Madeira, Campus da Penteada, 9020-105 Funchal, Portugal , Tel: +351 291705122; E-mail: vpereira@uma.pt

\begin{abstract}
Biogenic amines are naturally present in grapes or can occur during the vinification and aging processes, essentially due to the microorganism's activity. When present in wines in high amount, biogenic amines may cause not only organoleptic defects but also adverse effects in sensitive human individuals, namely due to the toxicity of histamine, tyramine and putrescine. Even though there are no legal limits for the concentration of biogenic amines in wines, some European countries only recommend maximum limits for histamine. In this sense, biogenic amines in wines have been widely studied. The determination of amines in wines is commonly achieved by liquid chromatography, using derivatization reagents in order to promote its separation and detection. In alternative, other promising methodologies have been developed using capillary electrophoresis or biosensors, revealing lower costs and faster results, without needing a derivatization step. Nowadays, it is still a challenge to develop faster and inexpensive techniques or methodologies to apply in the wine industry. Thus, this review will be focused on the studies published in the last decade that involves the determination of biogenic amines in wines, highlighting the novelty, improvement and optimization of the analytical methods. The sample preparation procedures (such as derivatization reagents), the analytical methodologies and the new trends being followed by the wine industry are also described and discussed.
\end{abstract}

Received Date: January 01, 2017

Accepted Date: February 13, 2017

Published Date: February 17, 2017

Citation: Pereira, V., et al. Analytical Methodologies for the Determination of Biogenic Amines in Wines: An Overview of the Recent Trends. (2017) J Anal Bioanal Sep Tech 2(1): 52- 57.

DOI: $10.15436 / 2476-1869.17 .1296$

Keywords: Biogenic amines; Wine; Chromatography; Derivatization; Capillary electrophoresis; Biosensors

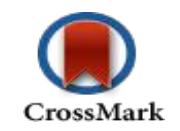

\section{Introduction}

Biogenic Amines (BAs) are nitrogenous low molecular weight compounds, naturally synthesized in plants, animals and microorganisms, with an important role on physiological functions, such as the regulation of body temperature and the secretion of gastric acid ${ }^{[1,2]}$. These compounds are essentially formed by decarboxylation of free amino acids by the activity of yeasts or bacteria, through substrate-specific enzymes, in a wide variety of protein-rich foods ${ }^{[1,3]}$. Also, other enzymatic reactions such as transamination, reductive amination and degradation of some precursors of amino compounds can also produce BAs. The knowledge about BAs in food and beverage's gaining interest in the lasts years, not only to ensure the quality of the products, since that they have been used as an indicator of spoilage, but mostly to guarantee the consumer protection, due to the potential toxic effect of BAs to humans ${ }^{[2]}$.
Wine is one of the most consumed fermented beverages in the world. Due to quality control and the growing consumers protection demands, the presence of BAs in wines has been largely studied ${ }^{[4]}$. Among the 25 different amines described in wines, the main ones are histamine, putrescine, tyramine and cadaverine $^{[5]}$ (Figure 1). Others are phenyl ethylamine, spermine, spermidine, agmatine and tryptamine. The presence of BAs in wines is frequent because these compounds can be naturally present in grapes and, therefore, its concentration depends on the grape variety, grape-growing conditions (climate, soil type, soil composition and fertilization, degree of maturation, etc.) and enological practices (skin maceration, $\mathrm{SO}_{2}$ concentration, vinification conditions, amino acid levels, clarification treatments, microorganism strains and aging processes) $)^{[2,6-11]}$. However, some BAs, such as putrescine and cadaverine, are considered indicators of poor sanitary conditions of grapes or lack of hygiene during winemaking ${ }^{[4,12]}$. 


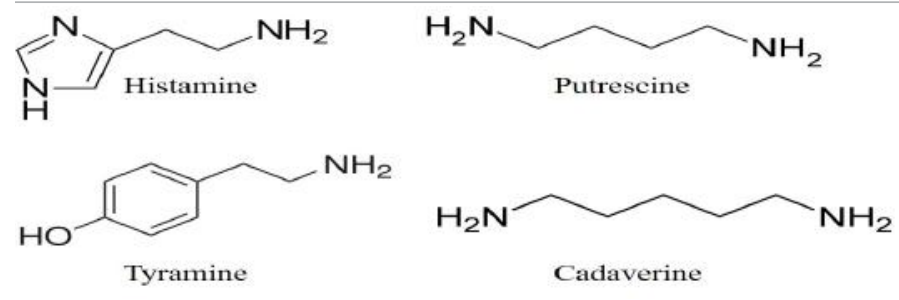

Figure 1: Chemical structure of the main biogenic amines found in wines.

The BAs found in grapes and musts are usually present in low concentrations, since they are produced and then degraded during winemaking. The fermentative stages (alcoholic and mostly malolactic) and the aging in barrels are the main winemaking processes that mostly contribute for their occurrence in wines. There are only some bacteria or yeast strains, carrying a specific metabolic pathway, that succeed to convert amino acid precursors into BAs and for that reason wines may contain different amounts of amines ${ }^{[13]}$. The BAs levels usually depend on the type of wine. For instance, red wines present higher levels of BAs than white wines, reaching levels up to $130 \mathrm{mg} / \mathrm{L}$ in Spanish red wines ${ }^{[14]}$. This fact is mainly due to the maceration process with grape skins, usually performed in red wine vinification, which promotes the increase of amino acids and elevates the fermentation temperature. On the contrary, generally, the malolactic fermentation is not applied or has a short duration in the white wine production ${ }^{[10,13]}$.

High amounts of BAs in wine may cause organoleptic and toxicological effects. Higher levels of putrescine and cadaverine can promote a negative flavor in wines, associated essentially with rancid notes ${ }^{[15]}$. On the other hand, histamine and tyramine are considered the main monoamines responsible for toxicity on wine consumers, since can promote symptoms similar to allergic reactions, namely gastrointestinal, cardiac, cutaneous and also nervous effects ${ }^{[16]}$. These effects occur mainly in susceptible individuals due to the presence of ethanol, which inhibit or reduce the activity of the enzymes responsible for the metabolism of BAs (monoamine and diamine oxidase) ${ }^{[17]}$. Thus, even though legal limits for BAs in wines are not yet established, some European countries have recommended maximum limits for histamine in wines, namely Germany $(2 \mathrm{mg} / \mathrm{L})$, France $(8$ $\mathrm{mg} / \mathrm{L})$ and Belgium $(6 \mathrm{mg} / \mathrm{L})^{[18]}$. Switzerland was the only country that had established an official upper limit of $10 \mathrm{mg} / \mathrm{L}$, but in 2011 it was removed for imported wines ${ }^{[19]}$.

The consumer safety, the wine quality control and the applied legislation are the main priorities for the wine industry. In order to facilitate the international trade, the International Organization of Vine and Wine (OIV) standardized the analytical methods and published two chromatographic methods for the determination of $\mathrm{BAs}^{[20]}$. However, even today, it is still a challenge the development of a simple, fast, low cost and reliable method for the determination of BAs in wines. In this sense, this review provides an overview of the recent advances observed in the analytical methodologies used for the determination of BAs in wines in the last decade, focusing the improvements, the optimization and the novelties in this field.

\section{Analytical methodologies}

The determination of BAs in wines has been a challenge for the scientific community due to their low concentration, complex matrices, the lack of chromophores, strong polarity or even the presence of structurally similar compounds ${ }^{[21]}$. Typically, the determination of BAs relies on liquid chromatography (LC), gas chromatography (GC) and capillary electrophoresis (CE). The detection is usually performed by diode array detection in the ultraviolet-visible region (DAD), fluorescence (FLD) and mass spectrometry (MS) ${ }^{[22-30]}$. Additionally, Sensors, Enzymatic and Immunoassays procedures as well as commercial test kits are already available, for the rapid detection of histamine and tyramine in wines $^{[31]}$.

Table 1: Chromatographic methodologies for the determination of biogenic amines in wine published in the last decade.

\begin{tabular}{|l|l|l|l|l|l|l|}
\hline $\begin{array}{l}\text { Chromatographic } \\
\text { technique }\end{array}$ & $\begin{array}{l}\text { Sample } \\
\text { pretreatment }\end{array}$ & $\begin{array}{l}\text { Derivatization } \\
\text { reagent }\end{array}$ & Matrix & Amines & Detection & Ref \\
\hline RP-HPLC & PVPP & DNS-Cl & Wine & His, Tyr, Put, Cad, Phe, Spm, Spmd & FLD \\
\hline RP-HPLC & PVPP & DNS-Cl & Wine & His, Tyr, Put, Cad, Phe, Spm, Spmd & UV-Vis & [32] \\
\hline RP-HPLC & PVPP & RTIL- DNS-Cl & Wine & Tyr, Put, Cad, Try, Phe, Spm & FLD & [35] \\
\hline TLC & PVPP & DNS-Cl & Wine & His, Tyr, Put, Cad & Densitometry & [40] \\
\hline RP-HPLC & - & OPA & Wine and honey & His, Tyr, Cad, Try, Phe, Isope & FLD & [29] \\
\hline RP-HPLC & - & OPA & Wine & His, Tyr, Put, Cad, Met, Try, Eth, Ety & FLD & [41] \\
\hline RP-HPLC & $\begin{array}{l}\text { N liquid and } \\
\text { TCA (10\%) }\end{array}$ & BzCl & $\begin{array}{l}\text { Wine, salami, } \\
\text { late and tuna, choco- }\end{array}$ & $\begin{array}{l}\text { His, Tyr, Put, Cad, Try, Spm, Spmd, } \\
\text { 3-Mtpa, Mpa, 3-Mba, 2-Mba, }\end{array}$ & MS/MS & [45] \\
\hline $\begin{array}{l}\text { RP-HPLC } \\
\text { (core-shellcolumn) }\end{array}$ & - & AQC & Wine & His, tyr, Put, Cad & FLD \\
\hline RP-HPLC & - & DEEMM & Wine & $\begin{array}{l}\text { His, Tyr, Put, Cad, Phe, Spm, Spmd, } \\
\text { Agm, Eth, Ser }\end{array}$ & UV-Vis & [34] \\
\hline RP-HPLC & - & FNBT & Wine & $\begin{array}{l}\text { Wine, salami, cheese, } \\
\text { tuna, anchovies and } \\
\text { olives }\end{array}$ & $\begin{array}{l}\text { His, Tyr, Put, Cad, Agm, Spm, Spmd, } \\
\text { Tea, Tma }\end{array}$ & CD \\
\hline IEC & PVPP & - & Wine and grape juice & 22 BAs & U21] \\
\hline GC & Centrifugation & IBCF & [46] \\
\hline
\end{tabular}

Technique: RP-HPLC: Reversed-Phase High Performance Liquid Chromatography; TLC: Thin-Layer Chromatography; IEC: Ion-Exchange Chromatography; GC: Gas Chromatography. 
Chemicals: PVPP: Polyvinylpyrrolidone; TCA: Trichloroacetic Acid. Derivatization agents: DNS-Cl: Dansyl Chloride; OPA: O-Phthalaldehyde; BzCl: Benzyl Chloride; AQC: 6-Aminoquinolyl-N-Hydroxysuccinimidyl Carbamate; DEEMM: Diethyl Ethoxymethylenemalonate; FNBT; 1-Fluoro-2-Nitro-4-(trifluoromethyl)Benzene; IBCF: Isobutyl Chloroformate.

Detection: UV-Vis: Ultraviolet-Visible Spectroscopy; FLD: Fluorescence Detector; MS: Mass Spectrometry; CD: Conductimetric Detector.

Amines: His: Histamine; Tyr: Tyramine; Put: Putrescine; Cad: Cadaverine; Met: Methylamine; Agm: Agmatine; Phe: Phenylethylamine; Spm: Spermine; Spmd: Spermidine; Try: Tryptamine; Ety: Ethylamine; Ser: Serotonin; Eth: Ethanolamine; Mpa: Methylpropylamine; 3-Mba: 3-Methylbutylamine; 2-Mba: 2-methylbutylamine; 3-Mtpa: 3-(Methylthio)propylamine; Isope: Isopentylamine; Tea: Triethylamine; Tma: Trimethylamine.

\section{Chromatographic methods}

In wines, the most commonly employed technology for the determination of BAs is the LC with Reversed-Phase (RP) separation, using C18 columns. Table 1 summarizes the chromatographic methods used to quantify BAs in wine, in the last decade. Typically the wine samples are analyzed directly or after a simple treatment with Polyvinylpolypyrolidone (PVPP) to remove some phenolic compounds ${ }^{[17-32]}$. Nowadays, most methodologies usually involve sample preparation prior to analysis, not only to remove some compounds that may interfere with the analysis but also to concentrate the analytes. Also, pre-column or post-column derivatization is often needed for appropriate detection, since BAs do not have enough absorption in the UVVis or FLD wavelength ranges. The derivatization step is also used to improve the separation in the RP columns, reducing the polarity of the original compounds ${ }^{[17,26,28,29,32-36]}$. Several derivatization reagents have been reported ${ }^{[37]}$ however, dansyl chloride (DNS-Cl) has been commonly used in the last years, since that its derivatives can be detected using DAD, FLD and $\mathrm{MS}^{[36]}$.

The determination of dansylated amines in wines has been the target of several studies ${ }^{[37,38]}$. The derivatization and chromatographic conditions have been optimized using the central composite designs, with BAs being detected by fluorescence $^{[32]}$ or UV-Vis ${ }^{[17]}$. FLD revealed better sensitivity to detect dansylated amines ${ }^{[17,32]}$. DNS-Cl has been largely used as pre-column derivatizing agent in the determination of BAs in wines and it produces stable derivatized compounds. However, the dansylation reaction is a time consuming process that requires the application of external temperature: 10 to $60 \mathrm{~min}$ at 40 to $70{ }^{\circ} \mathrm{C}^{[38,39]}$. Jiang, et al. ${ }^{[35]}$ introduced a new method to perform the dansylation at room temperature, during $20 \mathrm{~min}$, using ionic liquids as media for the derivatization of BAs in wines. A low-cost method based on thin layer chromatography using densitometry to quantify the dansylated BAs was also developed ${ }^{[40]}$ and it can be used for routine analysis of histamine, tyramine, putrescine and cadaverine in wine.

Ortho-Phthalaldehyde (OPA) is another recognized reagent for the derivatization of biogenic amines. OPA is one of the most used derivatization reagent and for that reason several studies report its use for the determination of BAs in wines. OPA derivatives are less stable but the reaction can occur at room temperature in a short time ${ }^{[41-43]}$. Pereira, et al. ${ }^{[29]}$ proposed a methodology using a pre-column derivatization with OPA, performed in $3 \mathrm{~min}$ into the sample injection loop of the HPLCFLD system, for the simultaneous determination of BAs and amino acids in wine, without using any preliminary separation or clean-up. The OIV also propose OPA as derivatization reagent for the BAs evaluation in musts and wines using HPLC-FLD ${ }^{[20]}$. In alternative, they also propose a methodology that uses Diethyl ethoxymethylenemalonate (DEEMM) to derivatize the BAs present in wine when it is used an HPLC-DAD system ${ }^{[20]}$. Also, Wang, et al. ${ }^{[44]}$ reported the development of a pre-column derivatization method with DEEMM for the quantification of $10 \mathrm{BAs}$ and 23 amino acids, using RP-HPLC-DAD, shortening the analysis time for $30 \mathrm{~min}$. This pre-column derivatizing agent enables the quantitative determination of secondary and primary amines, with no side-reaction products and produces stables derivatives at room temperature for several days, which can be detected by an UV detector, usually available in most chromatography laboratories ${ }^{[45]}$. Recently, a new derivatization reagent, namely 1-fluoro-2-nitro-4-(trifluoromethyl)benzene (FNBT), was also used for the determination of histamine, tyramine, tryptamine and phenyl ethylamine in wines using RP-HPLC-DAD analysis, showing simple and less-time consuming derivatization when compared to other methods ${ }^{[21]}$.

On the other hand, the combination of a core-shell C18 column with 6-Aminoquinolyl-N-Hydroxysuccinimidyl Carbamate (AQC) derivatization reagent, using FLD detection, was studied by Berbegal, et $\mathrm{al}^{[33]}$. They reported that this combination enables the analysis of BAs in wine reducing the run time and the use of organic solvents. Stable isotope dilution assays, with 10 isotopically labeled BAs as internal standards, have also been used for the quantification of BAs in several food matrices, including wine, with benzyl chloride $(\mathrm{BzCl})$ as derivatization agent using liquid chromatography with tandem mass spectrometric detection (LC-MS/MS $)^{[45]}$.

Ion-exchange chromatography combined with a conduct metric detector (IEC-CD) was successfully applied to determine BAs in wines. The LODs was not lower than those obtained by RP separation, however this method has the advantage that BAs do not need derivatization to be separated and detect$\mathrm{ed}^{[46]}$.

Finally, a gas chromatography-mass spectrometry (GC-MS) method was also used for the quantification of volatile and nonvolatile biogenic amines in Port wines, using Isobutyl Chloroformate (IBCF) as derivatization agent. Even though the method implies a complex procedure, it provides an accurate identification and enables the quantification of a higher number of BAs compared to the typical LC methods ${ }^{[24]}$.

\section{Electrophoretic methods}

Capillary electrophoresis (CE) is also used as a separation technique for the determination of BAs in wine (Table 2 ), with the advantage of being rapid and effective, with low reagent consumption. Automated on-line combination of capillary isotachophoresis-capillary zone electrophoresis (cITPCZE) with UV detection was successfully used for determination of selected BAs in wines, without a derivatization step ${ }^{[47]}$. The authors concluded that this method can be more sensitive for the determination of BAs in wines when comparing to other chromatographic and electrophoretic methods, due to the online pre-concentration of selected analytes in the cITP step, the high separation efficiency of the CZE and the fact that BAs can be detected by selective photometric detection, without needing derivatization. Another Isotachophoresis (cITP) method was 
proposed by Jastrzebska, et al ${ }^{[48]}$. After a simple sample preparation, without needing derivatization, they determined a group of important BAs in wines, in a 14-min run. The cITP method revealed to be an attractive choice comparatively to an HPLC method based on a pre-column derivatization with DNS-Cl.

Table 2: Electrophoretic methodologies for the determination of biogenic amines in wines published in the last decade.

\begin{tabular}{|c|c|c|c|c|c|c|}
\hline $\begin{array}{l}\text { Electrophoretic } \\
\text { technique }\end{array}$ & $\begin{array}{l}\text { Sample } \\
\text { pretreatment }\end{array}$ & $\begin{array}{l}\text { Derivatization } \\
\text { reagent }\end{array}$ & Matrix & Amines & Detection & Ref \\
\hline CITP & - & - & Wine and Beer & His, Tyr, Put, Cad, Phe, Try, Spm, Spmd & $\mathrm{CD}$ & {$[48]$} \\
\hline CITP/CZE & - & - & Wine & His, Tyr, Phe & UV-Vis & [47] \\
\hline $\mathbf{C E}$ & PVPP & - & Wine and Beer & His, Tyr, Put, Cad, Spm, Spmd, Try, Phe, Uro & MS/MS & [25] \\
\hline MECK & - & FITC & Wine & His, Tyr, Put, Cad, Spmd, Try, Phe & LIF & [57] \\
\hline
\end{tabular}

Technique: CE: Capillary Electrophoresis; CITP: Capillary Isotachophoresis; CZE: Capillary Zone Electrophoresis; MECK: Nonionic Micellar Electrokinetic chromatography.

Chemicals: PVPP: Polyvinylpyrrolidone.

Derivatization agent: FITC: Fluorescein IsothioCyanate.

Detection: CD: Conductimetric Detector; UV-Vis: Ultraviolet-Visible Spectroscopy; MS/MS: Tandem Mass Spectrometry; LIF: Laser-Induced Fluorescence.

Amines: His: Histamine; Tyr: Tyramine; Put: Putrescine; Cad: Cadaverine; Phe: Phenylethylamine; Spm: Spermine; Spmd: Spermidine; Try: Tryptamine; Uro: Urocanic acid.

Capillary electrophoresis-tandem mass spectrometry (CE-MS/MS) was also applied for the eco-friendly, simple and fast determination of BAs in wines ${ }^{[25]}$. The electrophoretic separation took just $10 \mathrm{~min}$ for the migration of $9 \mathrm{BAs}$ and the wine samples were just submitted to a PVPP clean-up and filtrated prior to analysis.

Finally, nonionic micellar electro kinetic chromatography with laser-induced fluorescence detection (MEKC-LIF) was used for the fast quantification (less than $9 \mathrm{~min}$ ) of 7 BAs in wine samples ${ }^{[49]}$ The MEKC-LIF method used Fluorescein Isothiocyanate (FITC) as derivatization reagent.

\section{Other methodologies}

In alternative to chromatography and electrophoresis, other techniques have been used for the BAs determination in wines, namely sensors and flow-injection analysis.

The development of biosensors has recently gained much interest among the scientific community because these low-cost devices can give results in a few minutes, without needing any kind of sample pre-treatment and the possibility to be used outside the laboratory. BAs biosensors, resulting from the combination of different enzymes for the bio-recognition of the BAs, are then a good option for a rapid determination of these compounds in wine ${ }^{[50,51]}$. The most used signal transducers of these biosensors are electrochemical sensors, which are generally based on the fluorescence response between BAs and sensor molecules interactions. Henao-Escobar, et al. ${ }^{[52]}$ produced a dual enzymatic sensor for the simultaneous determination of histamine and putrescine by measuring the oxidation current and Di Fusco, et al. ${ }^{[51]}$ characterized and evaluated the use of a diamine oxidase (DAO) from Lathyrus Sativus, as a biocatalytic component of an electrochemical biosensor, for the determination of the total amount of BAs in wines. On the other hand, Basozabal, et al. ${ }^{[53]}$ developed a sensor based on molecular imprinted nanoparticles with high affinity for histamine. Table 3 summarizes the use of biosensors for the determination of BAs in wines.

Table 3: Recent studies using biosensors for the determination of biogenic amines in wines.

\begin{tabular}{|l|l|l|l|l|l|}
\hline Type of biosensor & Amines & Sample pre-treatment & Recognition element & LOD \\
\hline Potentiometric sensor & Hist & $\begin{array}{l}\text { pH 5 }(\mathrm{NaOH}) \text { and then diluted (1:5 } \\
\text { or 1:10) in Ac buffer 10 mM (pH 5). }\end{array}$ & Molecularly imprinted nanoparticles & $1.12 \mu \mathrm{M}$ & {$[53]$} \\
\hline $\begin{array}{l}\text { Electrochemical sensor } \\
\text { (amperometry) }\end{array}$ & Hist, Put & Activated carbon & $\begin{array}{l}\text { Histamine deshydrogenase and } \\
\text { putrescine oxidase enzymes }\end{array}$ & $\begin{array}{l}8.1 \text { for His and } \\
10 \mu \mathrm{M} \text { for Put. }\end{array}$ & {$[52]$} \\
\hline $\begin{array}{l}\text { Electrochemical sensor } \\
\text { (amperometry) }\end{array}$ & Total BAs & $\begin{array}{l}\text { 0.1 M phosphate and adjusted the } \\
\text { pH for 7.4 with NaOH }\end{array}$ & Diamine oxidase from L. sativus & $200 \mu \mathrm{g} / \mathrm{L}$ & {$[51]$} \\
\hline
\end{tabular}

His: Histamine; Put: Putrescine; BAs: Biogenic amines

Although the enzyme-linked immunosorbent assay (ELISA) presents high sensitivity and precision, in the last decade, no studies were found in literature reporting this procedure for determining BAs in wines. Indeed, this methodology is time-consuming and may be expensive for a small number of samples ${ }^{[54]}$. The last report was made by Marcobal, et al. ${ }^{[55]}$, in 2005, which consisted in the development of a direct ELISA immunoassay method, specific for the determination of histamine in wines.

Finally, a flow injection method for the rapid determination of histamine in wine was reported by Hernández-Cassou, et al. ${ }^{[56]}$. This method was based on the reaction of histamine with 1,2-naphthoquinone-4-sulfonate to form a derivative that can be detected by a UV-Vis spectrophotometer ${ }^{[57]}$. The procedure is simple and can be automatized and miniaturized.

\section{Conclusion}

In the last decade, several methods for the determination of BAs in wines have been developed, in order to improve the precision and sensitivity. Most procedures use liquid chromatography and DNS-Cl as derivatizing reagent. However, oth- 
er reagents have been studied and are showing very promising results, regarding the time-consuming for sample preparation, analysis and also lower solvent consumption. The new trend for the determination of BAs in wines will go through portable, faster and cheaper methodologies to meet the demands of the wine industry. Thus, the development of biosensors has been gaining interest. Similarly, CE also demonstrates lower costs and faster results, without using the derivatization step.

\section{Acknowledgments}

Andreia Miranda, João M. Leça and Vanda Pereira are thankful to the Agência Regional para o Desenvolvimento da Investigação Tecnologia e Inovação (ARDITI) under the Project M1420 - 09-5369-FSE-000001 for the financial support through their grants.

\section{References}

1. Santos, M.H.S. Biogenic amines: their importance in foods. (1996) Int J Food Microbiol 29(2-3): 213-231.

Crossref

2. Shalaby, A.R. Significance of biogenic amines to food safety and human health. (1996) Food Res Int 29(7): 675-690.

Crossref

3. Sebastian, P., Herr, P., Fischer, U., et al. Molecular identification of lactic acid bacteria occurring in must and wine. (2011) S Afr J Enol 32(2): 300-309.

Crossref

4. Del Prete, V., Costantini, A., Cecchini, F., et al. Occurrence of biogenic amines in wine: The role of grapes. (2009) Food Chem 112(2): 474-481.

Crossref

5. Brink, B., Damink, C., Joosten, H.M.L.J., et al. Occurrence and formation of biologically active amines in foods. (1990) Int J Food Microbiol 11(1): 73-84.

Crossref

6. Cecchini, F., Morassut, M. Effect of grape storage time on biogenic amines content in must. (2010) Food Chem 123(2): 263-268.

Crossref

7. Herbert, P., Cabrita, M.J., Ratola, N., et al. Relationship between biogenic amines and free amino acid contents of wines and musts from Alentejo (Portugal). (2006) J Environ Sci Health B 41(7): 1171-1186.

Pubmed I Crossref

8. Hernández-Orte, P., Lape-a, A.C., Pe-a-Gallego, A., et al. Biogenic amine determination in wine fermented in oak barrels: Factors affecting formation. (2008) Food Res Int 41(7): 697-706.

Crossref

9. Izquierdo Ca-as, P.M., García Romero, E., Gómez Alonso, S., et al. Amino acids and biogenic amines during spontaneous malolactic fermentation in Tempranillo red wines. (2008) J Food Comp Anal 21(8): 731-735.

Crossref

10. Martín-Álvarez, P.J., Marcobal, Á., Polo. C., et al. Influence of technological practices on biogenic amine contents in red wines. (2006) Eur Food Res Technol 222(3): 420-424.

Crossref

11. Vidal-Carou, M.C., Codony-Salcedo, R., Mariné-Font, A. Histamine and tyramine in spanish wines: Relationships with total sulfur dioxide level, volatile acidity and malolactic fermentation intensity. (1990) Food Chem 35(3): 217-227.

Crossref

12. Leitão, M.C, Marques, A.P., San Romão, M.V. A survey of biogenic amines in commercial Portuguese wines. (2005) Food Control 16(3): 199-204.

Crossref
13. Lonvaud-Funel, A. Biogenic amines in wines: role of lactic acid bacteria. (2001) FEMS Microbiol Lett 199(1): 9-13.

Pubmed I Crossref

14. Marcobal, Á., Martín-Álvarez, P.J., Polo, M.C., et al. Formation of biogenic amines throughout the industrial manufacture of red wine. (2006) J Food Protect 69(2): 397-404.

Pubmed I Crossref

15. Antonio Palacios, C.S., Sibylle, K., Didier, T., et al. Wine quality and malolactic fermentation: Perception by wine drinkers of sensory defects caused by uncontrolled malolactic fermentation. (2004) Porto Crossref

16. Hazards, E.P.B. Scientific opinion on risk based control of biogenic amine formation in fermented foods. (2011) EFSA Journal 9(10): 2393.

\section{Crossref}

17. Henríquez-Aedo, K., Vega, M., Prieto-Rodríguez, S., et al. Evaluation of biogenic amines content in chilean reserve varietal wines. (2012) Food Chem Toxicol 50(8): 2742-2750.

Pubmed I Crossref

18. Lehtonen, P. Determination of amines and amino acids in wine - A review. (1996) Am J Enol Vitic 47(2): 127-133.

Crossref

19. Martuscelli, M., Arfelli, G., Manetta, A.C., et al. Biogenic amines content as a measure of the quality of wines of Abruzzo (Italy). (2013) Food Chem 140(3): 590-597.

Pubmed I Crossref

20. OIV. Compendium of International Methods of Analysis of Wines and Musts. (2016) Paris

Crossref

21. Jastrzębska, A., Piasta, A., Kowalska, S., et al. A new derivatization reagent for determination of biogenic amines in wines. (2016) J Food Comp Anal 48: 111-119.

Crossref

22. Almeida, C., Fernandes, J.O., Cunha, S.C. A novel dispersive liquid-liquid microextraction (DLLME) gas chromatography-mass spectrometry (GC-MS) method for the determination of eighteen biogenic amines in beer. (2012) Food ControL 25(1): 380-388. Crossref

23. Arce, L., Ríos, A., Valcárcel, M. Direct determination of biogenic amines in wine by integrating continuous flow clean-up and capillary electrophoresis with indirect UV detection. (1998) [J Chromatogr A 803(1-2): 249-260.

Crossref

24. Cunha, S.C., Faria, M.A., Fernandes, J.O. Gas chromatographymass spectrometry assessment of amines in port wine and grape juice after fast chloroformate extraction/derivatization. (2011) J Agric Food Chem 59(16): 8742-8753.

Pubmed I Crossref

25. Daniel, D., dos Santos, V.B., Vidal, D.T.R, et al. Determination of biogenic amines in beer and wine by capillary electrophoresis-tandem mass spectrometry. (2015) ] Chromatogr A 1416: 121-128.

Pubmed I Crossref

26. Gómez-Alonso, S., Hermosín-Gutiérrez, I., García-Romero, E. Simultaneous HPLC analysis of biogenic amines, amino acids, and ammonium ion as amino enone derivatives in wine and beer samples. (2007) J Agric Food Chem 55(3): 608-613.

Pubmed I Crossref

27. Mafra, I., Herbert, P., Santos, L., et al. Evaluation of biogenic amines in some Portuguese quality wines by HPLC fluorescence detection of OPA derivatives. (1999) Am J Enol Vitic 50(1): 128-132. Crossref

28. Dugo, G., Vilasi, F., La Torre, G.L., et. Reverse phase HPLC/DAD determination of biogenic amines as dansyl derivatives in experimental red wines. (2006) Food Chem 95(4): 672-676.

Crossref 
29. Pereira, V., Pontes, M., Câmara, J.S., et al. Simultaneous analysis of free amino acids and biogenic amines in honey and wine samples using in loop ortho-phthalaldeyde derivatization procedure. (2008) IJ Chromatogr A 1189(1-2): 435-443.

Pubmed I Crossref

30. Soufleros, E.H., Bouloumpasi, E., Zotou, A., t al. Determination of biogenic amines in Greek wines by HPLC and ultraviolet detection after dansylation and examination of factors affecting their presence and concentration. (2007) Food Chem 101(2): 704-716.

Crossref

31. Simon-Sarkadi, L., Gelencsér, É., Vida, A. Immunoassay method for detection of histamine in foods. (2003) Acta Aliment Hung 32(1)

Crossref

32. Pineda, A., Carrasco, J., Pe-a-Farfal, C., et al. Preliminary evaluation of biogenic amines content in Chilean young varietal wines by HPLC. (2012) Food Control 23(1): 251-257.

Crossref

33. Berbegal, C., Pardo, I., Ferrer, S. The use of core-shell high-performance liquid chromatography column technology to improve biogenic amine quantification in wine. (2016) J Sci Food Agr 96(5): 1556-1561. Pubmed I Crossref

34. Hernández-Orte, P., Pe-a-Gallego, A., Ibarz, M.J., et al. Determination of the biogenic amines in musts and wines before and after malolactic fermentation using 6-aminoquinolyl-N-hydroxysuccinimidyl carbamate as the derivatizing agent. (2006) J Chromatogr A 1129(2): 160-164.

Pubmed I Crossref

35. Jiang, H-L., Ying, L-Y., Zhou, S-C., et al. Chromatographic determination of biogenic amines in wines after treatment with ionic liquids as novel media. (2011) J Sep Sci 34(9): 1055-1062.

Pubmed I Crossref

36. Pe-a-Gallego, A., Hernández-Orte, P., Cacho, J., et al. High-performance liquid chromatography analysis of amines in must and wine: A review. (2012) Food Rev Int 28(1): 71-96.

Crossref

37. Önal. A., Tekkeli, S.E.K., Önal, C. A review of the liquid chromatographic methods for the determination of biogenic amines in foods. (2013) Food Chem 138(1): 509-515.

Pubmed I Crossref

38. Bedia Erim, F. Recent analytical approaches to the analysis of biogenic amines in food samples. (2013)'Trends Anal Chem TrAC 52: 239247.

Crossref

39. Basheer, C., Wong, W., Makahleh, A., et al. Hydrazone-based ligands for micro-solid phase extraction-high performance liquid chromatographic determination of biogenic amines in orange juice. (2011) J Chromatogr A 1218(28): 4332-4339.

Pubmed I Crossref

40. Romano, A., Klebanowski, H., La Guerche, S., et al. Determination of biogenic amines in wine by thin-layer chromatography/densitometry. (2012) Food Chem 135(3): 1392-1396.

Pubmed I Crossref

41. Arrieta, M.P., Prats-Moya, M.S. Free amino acids and biogenic amines in Alicante Monastrell wines. (2012) Food Chem 135(3): 15111519.

Pubmed I Crossref

42. Kelly, M.T., Blaise, A., Larroque, M. Rapid automated high performance liquid chromatography method for simultaneous determination of amino acids and biogenic amines in wine, fruit and honey. (2010) $\mathfrak{\jmath}$ Chromatogr A 1217(47): 7385-7392.

Pubmed I Crossref

43. Meléndez, M.E., Sarabia, L.A., Ortiz, M.C. Distribution free methods to model the content of biogenic amines in Spanish wines. (2016) Chemometr Intell Lab 155: 191-199.

Crossref
44. Wang, Y-Q., Ye, D-Q., Zhu, B-Q., et al. Rapid HPLC analysis of amino acids and biogenic amines in wines during fermentation and evaluation of matrix effect. (2014) Food Chem 163: 6-15.

Pubmed I Crossref

45. Mayr, C.M., Schieberle, P. Development of stable isotope dilution assays for the simultaneous quantitation of biogenic amines and polyamines in foods by LC-MS/MS. J. Agric. (2012) Food Chem 60(12): 3026-3032.

Pubmed I Crossref

46. Palermo, C., Muscarella, M., Nardiello, D., et al. A multi residual method based on ion-exchange chromatography with conductivity detection for the determination of biogenic amines in food and beverages. 『(2013) Anal Bioanal Chem 405(2): 1015-1023.

Pubmed I Crossref

47. Ginterová, P., Marák, J., Staňová, A., et al. Determination of selected biogenic amines in red wines by automated on-line combination of capillary isotachophoresis-capillary zone electrophoresis. (2012) J Chromatogr B 904: 135-139.

Pubmed I Crossref

48. Jastrzębska, A., Piasta, A., Szłyk, E. Simultaneous determination of selected biogenic amines in alcoholic beverage samples by isotachophoretic and chromatographic methods. (2014) Food Addit Contam Part A. 31(1): 83-92.

Pubmed I Crossref

49. Başkan S, Tezcan F, Köse S, Öztekin N, Erim FB. Non-ionic micellar electrokinetic chromatography with laser-induced fluorescence: A new method tested with biogenic amines in brined and dry-salted fish. (2010) Electrophoresis 31(13): 2174-9.

Pubmed I Crossref

50. Kivirand K, Rinken T. Biosensors for biogenic amines: The present state of art mini-review. (2011) Anal Lett 44(17): 2821-33.

Crossref

51. Di Fusco, M., Federico, R., Boffi, A., et al. Characterization and application of a diamine oxidase from Lathyrus sativus as component of an electrochemical biosensor for the determination of biogenic amines in wine and beer. (2011) Anal Bioanal Chem 401(2): 707-716.

Pubmed I Crossref

52. Henao-Escobar, W., del Torno-de Román, L., Domínguez-Renedo, O., et al. Dual enzymatic biosensor for simultaneous amperometric determination of histamine and putrescine. (2016) Food Chem 190: 818823.

Pubmed I Crossref

53. Basozabal, I., Guerreiro, A., Gomez-Caballero, A., et al. Direct potentiometric quantification of histamine using solid-phase imprinted nanoparticles as recognition elements. (2014) Biosens Bioelectron 58: 138-144.

Pubmed I Crossref

54. Alves, R.C., Barroso, M.F., González-García, M.B., et al. New trends in food allergens detection: Toward biosensing strategies. (2016) Crit Rev Food Sci Nutr 56(14): 2304-2319.

Pubmed I Crossref

55. Marcobal, A., Polo, M.C., Martín-Álvarez, P.J., Moreno-Arribas MV. Biogenic amine content of red Spanish wines: comparison of a direct ELISA and an HPLC method for the determination of histamine in wines. (2005) Food Res Int 38(4): 387-394.

Crossref

56. Hernández-Cassou, S., Saurina, J. Determination of histamine in wine samples by flow-injection analysis and multivariate calibration. (2013) Anal Lett 46(11): 1758-1768.

Crossref

57. Uzaşçı, S., Başkan, S., Erim, F.B. Biogenic amines in wines and pomegranate molasses - a non-ionic micellar electrokinetic chromatography assay with laser-induced fluorescence detection. (2012) Food Anal Method 5(1): 104-108.

Crossref 